\title{
Biodiversity conservation for sustainable future
}

\author{
Naveen Kumar Arora ${ }^{1}$
}

(c) Society for Environmental Sustainability 2018

Existence of huge diversity of life forms is key feature of the blue planet. All the life forms ranging from bacteria to plants or animals play important roles on the only celestial body known to harbor life in the universe. These life forms are in dynamic relationship with one another. It is impossible for any life form to survive on earth without the direct or indirect support of other organisms. Biodiversity is thus a key feature of the planet earth and important for sustaining the ecosystems on it. Biodiversity is also exploited for various purposes by humans and helps in improving the quality of our lives. In fact the existence of human civilization is directly linked to the biodiversity of the planet. An ecosystem or habitat with high biodiversity is rich on biological wealth and is more important in terms of economy and ecological inputs. Abundant biodiversity is also an indicator of high levels of natural resources and balance of physicochemical and biological components. But there is a harsh reality which we are facing at present. The biodiversity loss is at its highest rate since the arrival of Homo sapiens on the blue planet. It is also believed that at present we are going through the sixth mass extinction of species on earth. The unfortunate part is that this mass extinction is scripted by humans. A species itself is resulting in wipeout of a large number of other life forms in the name of development and prosperity unknowing to the fact that it will result in unforeseen problems and lead to depletion of resources and endangering all life forms. Development in the present form is unsustainable and harmful for the life and soul of the planet.

Tropical forests and tropical oceans are amongst the richest biodiversity zones on earth. It is estimated that two-thirds of the biodiversity of earth is present in tropical regions. But as per studies and available data, tropical forests are depleting at the rate of $0.8 \%$ per annum. Earth has lost $10 \%$ of tropical forests in last 25 years due to human related

Naveen Kumar Arora

nkarora.bbau@gmail.com

1 Department of Environmental Science, BBA University, Lucknow, UP, India activities. The invaluable input of tropical forests just in the form of oxygen is estimated to be more than $25 \%$ and that of oceans as approximately $70 \%$ of the total (oxygen) produced on earth. Although tropical forests and oceans are resources for umpteen other natural products but a single input (oxygen) alone shows the immense importance of these habitats in survival of most of the life forms on earth. As per the estimates of World Wide Fund for Nature (WWF) report in 2014, global biodiversity declined by $52 \%$ from 1970 to 2010. Almost half of the land mammals have lost more than $80 \%$ of their range in last century. It is also estimated that $83 \%$ of wild mammals have already been lost due to human activities and if the situation continues almost all of the wild mammals on land will be wiped out very soon. Huge loss in avian, reptilian and amphibian biodiversity is also clearly visible. The major reasons for decline in biodiversity are loss of habitat, anthropogenic activities such as release of dangerous synthetic pollutants, increase in concentration of green house gases resulting in global warming and climate change, overexploitation of natural resources, spread of diseases and invasive species due to human interference. The mass extinction of species, also termed as "biological annihilation" by the scientists, is far quicker than was expected earlier.

The major biomass of soil and water bodies on earth are composed of microorganisms and it is estimated that 50 million bacterial cells are present in a gram of soil and in oceans approximately $90 \%$ of the total biomass is made up of microbes. Because of their ability to adapt metabolically and genetically to almost any conditions on the planet, microbes are present in every corner of the earth and are considered as the building blocks of the ecosystems. These little creatures which mainly include bacteria and fungi are by and large responsible for biological cycling of nutrients, biodegradation of wastes (natural or human generated), providing various vital services to plants and animals apart from being most commonly exploited as biological tools in industries. Hence it is important to study the diversity of these organisms in different habitats and ecosystems. However, it is almost impossible to estimate the diversity of microbes by present tools and techniques. It is even more difficult to 
determine or estimate the microbial species which have got extinct from the ecosystems. Some recent studies suggest that population of very important microbes such as symbiotic nitrogen fixing rhizobial species have declined drastically from the soils where chemical pesticides are being used continuously, as observable by decline in nodulation in legume crops. Similarly lichens are becoming extinct with increase in pollution levels of air and soil. Decline in soil organic matter also results in loss of microbial diversity. These are just a few visible examples of decline in population of useful microorganisms from ecosystems. Microorganisms are important to maintain the sustainability of the habitats and environment as whole, thus it becomes imperative to conserve the microbial diversity of the ecosystems.

Environmental sustainability index is in direct relation to the biological diversity. Hence there is an urgent need to protect the diversity so as to sustain the life on earth. Biodiversity conservation should be the top priority of all the nations. Although several protocols, regulations and agreements have been framed but still the targets have not been achieved. International Union for Conservation of Nature and Natural Resources (IUCN) is the global authority on the status of the natural world and the steps required to protect it. Its main goal is to provide forum and networking for the governments, non-government organizations, businesses, scientists and individuals throughout the globe to work for conservation of biodiversity, and achieve targets of sustainable development. International agreements such as Convention on Biological Diversity (CBD) and Convention for International Trade in Endangered Species (CITES), both involved in conservation of global biodiversity, are the offspring of IUCN. The main goals of CBD agreement at Rio de Janeiro in 1992 (which has 196 signatory countries) are; conservation of biodiversity, sustainable use of biodiversity and fair and equitable sharing of the benefits arising from the conservation of genetic resources. Several other agreements have been signed at the global level under CBD by most of the nations for conserving biodiversity which include, Cartagena Protocol on Biosafety 2000, The Nagoya Protocol on Access to Genetic Resources and Fair and Equitable Sharing of Benefits Arising from their Utilization (ABS) 2010 and Aichi Biodiversity Targets 2020. The IUCN Global Species Programme along with IUCN Species Survival Commission provides the current status of endangered and critically endangered species of plants, animals and fungi and information on their conservation status and distribution. Although all the agreements and programmes have set some regulations and targets, but it is important that the goals are achieved within the stipulated time and the success or failure of the protocols is monitored at the global and micro level. Time has come to unite and make serious efforts to conserve the biodiversity of earth otherwise the consequences will be far impacting and irreversible. The clock is ticking very fast and we have little time left to protect the civilization from complete wipe out.

Hard times call for drastic measures, and indeed as far as biodiversity is concerned the situation is alarming. There should be multiple inter-linked repositories of the biological information at international level. The countries should make sincere efforts to conserve critically endangered flora and fauna. Treaties should not only be signed but seriously enforced as well, particularly between the nations which share ecosystems and habitats of critically endangered organisms. Separate information centers and repositories should be created for the conservation of oceanic diversity. There can also be sub-centers for different oceans/seas at key locations as each one of them can involve several countries. Similarly diversity of microorganisms should also be studied and conserved. According to estimates we know only about less than $1 \%$ of microorganisms (of the total present in the ecosystems). Molecular microbial ecology is an upcoming discipline which involves the molecular tools and techniques to provide the knowledge of complex microbial communities in diverse habitats. These high through put approaches are used to provide information about known and unknown microbial community structures in an ecosystem. Metagenomic analysis has great potential in understanding the diversity of unculturable microbial populations and identification of novel genes of interest from the environment/nature. Integration of metagenomics, metatranscriptomics, and metaproteomics can be very useful in near future to study and predict the microbial diversity and its functions in an ecosystem. The need is to use these new generation technologies in natural habitats for determining the microbial abundance, dominant and autochthonous types, functions, and shifts in relation to natural and anthropogenic interferences. At present very few nations of the globe have truly functional microbial collection centers. In fact microbial diversity of several habitats and ecosystems is completely unknown. This will also help in finding novel microbes and genes for the industry and biotechnological applications. The microbes are performing bulk of the metabolic and genetic activities on earth and have the biggest and most diverse genetic pool as well. Understanding of microbial diversity will result in understanding the genetics and metabolic working of the ecosystems in a holistic manner.

It is also important to have the diversity information repositories or centers for fragile habitats such as the Arctic and Antarctic regions, Himalayas, tropical rain forests, tropical oceanic zones, to name a few. These delicate but 
very important ecosystems are not the heritage of particular nations only and need to be looked after by all in a very collective manner. Biodiversity conservation is the responsibility and obligation of every citizen of the globe so as to handover a lively planet to future generations. Of course biodiversity conservation is the key focus area of the journal "Environmental Sustainability".

Naveen Kumar Arora

Editor in Chief 